\title{
Bifurcation Analysis of a Fractional-Order Delayed Rolling Mill's Main Drive Electromechanical Coupling System
}

\author{
Rui Zhang, ${ }^{1}$ Jinbin Wang $\mathbb{D}^{1,2,3}$ and Lifeng $M a \mathbb{D}^{2,3}$ \\ ${ }^{1}$ School of Applied Science, Taiyuan University of Science and Technology, China \\ ${ }^{2}$ School of Mechanical Engineering, Taiyuan University of Science and Technology, China \\ ${ }^{3}$ Engineering Research Center Heavy Machinery Ministry of Education, Taiyuan University of Science and Technology, China
}

Correspondence should be addressed to Jinbin Wang; wangjinbin@tyust.edu.cn and Lifeng Ma; malifengasd@163.com

Received 12 June 2021; Revised 21 August 2021; Accepted 25 August 2021; Published 11 September 2021

Academic Editor: Zine El Abiddine Fellah

Copyright @ 2021 Rui Zhang et al. This is an open access article distributed under the Creative Commons Attribution License, which permits unrestricted use, distribution, and reproduction in any medium, provided the original work is properly cited.

This work is focused on a rolling mill's main drive electromechanical coupling system. Firstly, we equip electromechanical coupling system with fractional-order time delay. Secondly, we, respectively, derive the conditions for occurrence of Hopf bifurcation around equilibriums $E_{0}(0,0,0,0)$ and $E_{1}\left(x_{1}^{*}, 0, x_{3}^{*}, 0\right)$. It is found that the fractional order $\alpha$ and time delay $\tau$ in the system play an important role on the system stability. Finally, numerical simulations are given to verify the analytic results.

\section{Introduction}

The rolling mill system, as a typical complex nonlinear system, involves many disciplines such as rolling, machines, motors, and automatic control. Due to the complex structure of the rolling mill, the system contains a variety of nonlinear factors, which closely affect the intensity and frequency of torsional vibration during the production process of the rolling mill. Frequent torsional vibrations during rolling mill production have a serious impact on the quality of rolled products. The phenomenon of torsional vibration not only causes vibration marks on the surface of the strip and rolls and reduces the service life of the rolls but also deteriorates the operating environment, shortens the fatigue life of parts, and ultimately threatens the safety of the rolling mill. Therefore, being able to propose effective measures to suppress the torsional vibration of the rolling mill [1-10] has become the key to solving the torsional vibration of the main drive of the rolling mill. At the same time, this issue has also attracted scholars' attention.

Rotating machinery systems contain various nonlinear factors, which lead to complex dynamic behaviors such as Hopf bifurcation and chaos of the system (see [11-13]).
The researchers [14-16] abstracted the main drive system of the rolling mill as a two-mass or multimass relative rotation system and studied the bifurcation and chaos of the torsional vibration of the system. In our previous work [17], we designed a nonlinear controller to control the Hopf bifurcation in the main drive delayed system of the rolling mill.

In 2015, Liu et al. [16] deduced a nonlinear electromechanical coupling system by using the dissipation Lagrange equation, and they introduced a time delay feedback to control the dynamic behaviors of the system. The model takes the following form:

$$
\left\{\begin{array}{l}
J_{1} \ddot{\varphi}_{1}+K\left(\varphi_{1}-\varphi_{2}\right)+C\left(\dot{\varphi}_{1}-\dot{\varphi}_{2}\right)+C_{e} \dot{\varphi}_{1}-K_{e} \varphi_{1}=k_{0}+k_{2} \varphi_{1}^{2}+k_{3} \varphi_{1}^{3} \\
J_{2} \ddot{\varphi}_{2}-K\left(\varphi_{1}-\varphi_{2}\right)-C\left(\dot{\varphi}_{1}-\dot{\varphi}_{2}\right)=T_{L}+g_{c 1} \varphi_{2}\left(t-\tau_{1}\right)+g_{c 2} \varphi_{2}^{3}\left(t-\tau_{2}\right)
\end{array},\right.
$$

where $J_{i}(i=1,2)$ is the moment of inertia, $\varphi_{i}(i=1,2)$ and $\dot{\varphi}_{i}(i=1,2)$ are the angle of rotation and rotational speed, respectively, $K$ is the torsional stiffness of drive shaft, $C$ is the shafting damping coefficient, and $C_{e}$ is the electromagnetic damping coefficient (the meaning of above parameters can refer to Ref. [16]). 
By applying appropriate transformations, system (1) is equivalent to system (2):

$$
\left\{\begin{array}{l}
\dot{x}_{1}=x_{2} \\
\dot{x}_{2}=-\omega_{1}^{2} x_{1}-b_{1} x_{2}+a_{1} x_{3}+d_{1} x_{4}+l_{1} x_{1}^{2}+l_{2} x_{1}^{3} \\
\dot{x}_{3}=x_{4} \\
\dot{x}_{4}=a_{2} x_{1}+d_{2} x_{2}-\omega_{2}^{2} x_{3}-b_{2} x_{4}+\beta_{1} x_{3}\left(t-\tau_{1}\right)+\beta_{2} x_{3}^{3}\left(t-\tau_{2}\right)
\end{array}\right.
$$

where $\omega_{1}^{2}=\left(K-K_{\mathrm{e}}\right) / J_{1} b_{1}=\left(C+C_{\mathrm{e}}\right) / J_{1}, a_{1}=K / J_{1}, d_{1}=C /$ $J_{1}, l_{1}=k_{2} / J_{1}, l_{2}=k_{3} / J_{1}, \omega_{2}^{2}=K / J_{2}, b_{2}=C / J_{2}, a_{2}=K / J_{2}, d_{2}=$ $C / J_{2}, \beta_{1}=g_{\mathrm{cl}} / J_{2}, \beta_{2}=g_{\mathrm{c} 2} / J_{2}$. At present, the stability and the Hopf bifurcation for system (2) were investigated in [16]. Ding et al. [18] focused on Hopf and Hopf-pitchfork bifurcations for system (2) by applying the multiple time scale method and the normal forms.

For electromechanical coupling system, the unstable bifurcation may cause a destructive vibration. Therefore, finding more efficient measures to control vibration is vital to system (2). In the existing literature, the electromechanical coupling systems are used ordinary differential equations or delay differential equations to investigate through the integer-order mathematical modeling. However, few studies have applied fractional order to system (2). At present, in the process of studying some real world problems, it is found that the problems can be better described by the fractionalorder models [19-21] than the integer-order models. Therefore, fractional-order systems may be the basis for many future studies. In [22-25], when introducing fractional orders to existing systems, the usual method is to directly replace the integer order with the fractional order. Motivated by the aforementioned works, we follow the same processing method and try to introduce the fractional order into system (2) and explore the effect of fractional order on the damping term of the system (1); the new model is described by the following fractional-order differential equations

$$
\left\{\begin{array}{l}
D^{\alpha} x_{1}=x_{2}, \\
D^{\alpha} x_{2}=-\omega_{1}^{2} x_{1}-b_{1} x_{2}+a_{1} x_{3}+d_{1} x_{4}+l_{1} x_{1}^{2}+l_{2} x_{1}^{3}, \\
D^{\alpha} x_{3}=x_{4} \\
D^{\alpha} x_{4}=a_{2} x_{1}+d_{2} x_{2}-\omega_{2}^{2} x_{3}-b_{2} x_{4}+\beta_{1} x_{3}\left(t-\tau_{1}\right)+\beta_{2} x_{3}^{3}\left(t-\tau_{2}\right),
\end{array}\right.
$$

where $D^{\alpha}$ represents the Caputo fractional derivative and $\alpha$ is the fractional and derivative order of damping, which may represent the nonlocal displacement effects of dissipation of energy (internal friction). $\tau_{1}$ and $\tau_{2}$ are time delays. The main contributions of this paper are as follows:

(1) A fractional-order nonlinear electromechanical coupling system with delay is proposed

(2) The conditions of Hopf bifurcation of system (3) around equilibrium are given
(3) Some simulations are implemented to corroborate the obtained conclusions

The layout of this work is organized as follows: In Section 2, the basic definition and the required theorem are introduced. In Section 3, stability analysis of fractionalorder nonlinear electromechanical coupling system with delay is obtained. In Section 4, some simulations are carried out to verify the theoretical results. Conclusions are given in Section 5.

\section{Preliminaries}

Before starting the analysis and establishing the results, we firstly introduce the basic definition and the required theorem.

Definition 1 [26]. The Caputo fractional derivative operator of order $\alpha(\alpha>0)$ is defined as

$\left(D_{0}^{\alpha} f\right)(x)= \begin{cases}\frac{d^{n} f(x)}{d x^{n}}, & \alpha=n \in N^{+}, \\ \frac{1}{\Gamma(n-\alpha)} \int_{0}^{x}(x-t)^{n-\alpha-1} f^{(n)}(t) d t, & 0<n-1 \leq \alpha \leq n,\end{cases}$

where $n$ is an integer, $x>0$.

Theorem 2 [19, 27] (the conditions of stability). Consider the following fractional-order system:

$$
D_{0}^{\alpha} x(t)=f(t), x(0)=x_{0}
$$

where $0<\alpha<1$. The equilibrium points of the above system are the solutions of $f(x)=0$. An equilibrium point is locally asymptotically stable if all eigenvalues $\lambda_{i}$ of the Jacobian matrix $J=\partial f / \partial x$ evaluated at the equilibrium point satisfy $\arg \left(\lambda_{i}\right) \mid>\alpha \pi / 2$. Further, if $\left|\arg \left(\lambda_{i}\right)\right|=\alpha \pi / 2$, then the system undergoes a Hopf bifurcation.

Theorem 3 [28, 29]. Consider the following $n$-dimensional fractional-order system with delay:

$$
D_{0}^{\alpha} x_{j}(t)=f_{j}\left(x_{1}(t), x_{2}(t), \cdots, x_{n}(t), \tau\right),(j=1,2, \cdots, n),
$$

where $0<\alpha<1$ and time delay $\tau \geq 0$. System (6) undergoes $a$ Hopf bifurcation at the equilibrium point $x^{*}=\left(x_{1}^{*}, x_{2}^{*}, \cdots, x_{n}^{*}\right)$ when $\tau=\tau_{0}$ if the following conditions are satisfied:

(i) All the eigenvalues $\lambda_{j}(j=1,2, \cdots, n)$ of the coefficient matrix $A$ of the linearized system of (7) with $\tau=0$ satisfy $\left|\arg \left(\lambda_{i}\right)\right|>\alpha \pi / 2$

(ii) The characteristic equation of the linearized system of (7) has a pair of purely imaginary roots $\pm \omega_{0}$ when $\tau=\tau_{0}$

(iii) $\left.\operatorname{Re}[d s(\tau) / d \tau]\right|_{\omega=\omega_{0}, \tau=\tau_{0}} \neq 0$ 


\section{Stability and Hopf Bifurcation}

Herein, the conditions of Hopf bifurcation of system (3) around feasible equilibrium points are mainly investigated. Noticing that system (3) has a zero equilibrium point $E_{0}(0$ $, 0,0,0)$ and a nonzero equilibrium point $E_{1}\left(x_{1}^{*}, 0, x_{3}^{*}, 0\right)$. The zero equilibrium point $E_{1}\left(x_{1}^{*}, 0, x_{3}^{*}, 0\right)$ satisfies the following equations:

$$
\left\{\begin{array}{l}
x_{2}=0 \\
-\omega_{1}^{2} x_{1}+a_{1} x_{3}+l_{1} x_{1}^{2}+l_{2} x_{1}^{3}=0, \\
x_{4}=0 \\
a_{2} x_{1}-\omega_{2}^{2} x_{3}+\beta_{1} x_{3}+\beta_{2} x_{3}^{3}=0 .
\end{array}\right.
$$

Then, by a direct computation, we can derive that $x_{1}$ sat-

$m_{1} x_{1}^{8}+m_{2} x_{1}^{7}+m_{3} x_{1}^{6}+m_{4} x_{1}^{5}+m_{5} x_{1}^{4}+m_{6} x_{1}^{3}+m_{7} x_{1}^{2}+m_{8} x_{1}+m_{9}=0 .\left\{\begin{array}{l}p_{1}(\omega) \cos \left(\omega \tau_{1}\right)-p_{2}(\omega) \sin \left(\omega \tau_{1}\right)+q_{1}(\omega)=0, \\ p_{2}(\omega) \cos \left(\omega \tau_{1}\right)+p_{1}(\omega) \sin \left(\omega \tau_{1}\right)+q_{2}(\omega)=0,\end{array}\right.$

The detailed expression of parameters $\left(m_{i}, i=1 \cdots 9\right)$ in Equation (8) can be found in Appendix A.

In order to meet the actual problem, we suppose that Equation (8) has a positive root $x_{1}^{*}>0$. Noticing the fact that $x_{3}=\left(1 / a_{1}\right) \omega_{1}^{2} x_{1}-l_{1} x_{1}^{2}-l_{2} x_{1}^{3}$, we can calculate $x_{3}^{*}$. Furthermore, if we suppose $x_{3}^{*}>0$, then system (3) has a nonzero equilibrium point $E_{1}\left(x_{1}^{*}, 0, x_{3}^{*}, 0\right)$.

Next, we, respectively, investigate the stability of system (3) around equilibrium points $E_{0}(0,0,0,0)$ and $E_{1}\left(x_{1}^{*}, 0, x_{3}^{*}\right.$ $, 0)$. In what follows, we divide the problem into two cases:

Case 1. Stability of $E_{0}=(0,0,0,0)$ with $\tau_{1} \geq 0, \tau_{2}=0$. (3) is

The characteristic equation of linear system of system

$$
s^{4 \alpha}+e_{1} s^{3 \alpha}+e_{2} s^{2 \alpha}+e_{3} s^{\alpha}+e_{4}+\left(f_{1} s^{2 \alpha}+f_{2} s^{\alpha}+f_{3}\right) \mathrm{e}^{-\tau_{1} s}=0,
$$

where $e_{1}=b_{1}+b_{2}, \quad e_{2}=b_{1} b_{2}+\omega_{1}^{2}+\omega_{2}^{2}-d_{1} d_{2}, \quad e_{3}=b_{2} \omega_{1}^{2}+$ $b_{1} \omega_{2}^{2}-d_{1} a_{2}-d_{2} a_{1}, e_{4}=\omega_{1}^{2} \omega_{2}^{2}-a_{1} a_{2}, f_{1}=-\beta_{1}, f_{2}=-b_{1} \beta_{1}$, and $f_{3}=-\beta_{1} \omega_{1}^{2}$.

For simplicity, Equation (9) is equivalent to

$$
P(s)+e^{-\tau_{1} s} Q(s)=0,
$$

where $P(s)=s^{4 \alpha}+e_{1} s^{3 \alpha}+e_{2} s^{2 \alpha}+e_{3} s^{\alpha}+e_{4}$ and $Q(s)=f_{1} s^{2 \alpha}$ $+f_{2} s^{\alpha}+f_{3}$.

When $\tau_{1}=0$, the characteristic Equation (10) at $E_{0}=$ $(0,0,0,0)$ becomes

$$
F(\lambda)=\lambda^{4}+h_{1} \lambda^{3}+h_{2} \lambda^{2}+h_{3} \lambda+h_{4},
$$

where $\lambda=s^{\alpha}, h_{1}=e_{1}, h_{2}=e_{2}+f_{1}, h_{3}=e_{3}+f_{2}$, and $h_{4}=e_{4}$ $+f_{3}$. Applying Routh-Hurwitz criterion for fractionalorder differential equations [30], one can deduce that fractional-order system (11) is asymptotically stable when $\tau_{1}=0$ if and only if

$(H 1): h_{i}>0(i=1,2,3,4), h_{1} h_{2}-h_{3}>0, h_{1} h_{2} h_{3}-h_{4} h_{1}^{2}-h_{3}^{2}=0$.

When $\tau_{1} \neq 0$, multiplying $e^{s \tau_{1}}$ on both sides of Equation (10), then one has

$$
P(s) e^{\tau_{1} s}+Q(s)=0
$$

By replacing $s=i \omega=(\cos (\pi / 2)+i \sin (\pi / 2)) \omega$ into Equation (13) and separating into its real and imaginary parts, we can obtain

where $P(i \omega)=p_{1}(\omega)+i p_{2}(\omega)$ and $Q(i \omega)=q_{1}(\omega)+i q_{2}(\omega)$. Then, by solving Equation (14), we can get the following

$$
\left\{\begin{array}{l}
\cos \left(\omega \tau_{1}\right)=\frac{-p_{1}(\omega) q_{1}(\omega)-p_{2}(\omega) q_{2}(\omega)}{p_{1}^{2}(\omega)+p_{2}^{2}(\omega)} \doteq H_{1}(\omega), \\
\sin \left(\omega \tau_{1}\right)=\frac{-p_{1}(\omega) q_{2}(\omega)+p_{2}(\omega) q_{1}(\omega)}{p_{1}^{2}(\omega)+p_{2}^{2}(\omega)} \doteq H_{2}(\omega),
\end{array}\right.
$$

where $\quad p_{1}(\omega)=\omega^{4 \alpha} \cos (2 \pi \alpha)+e_{1} \cos (3 \pi \alpha / 2) \omega^{3 \alpha}+e_{2} \omega^{2 \alpha}$ $\cos (\pi \alpha)+e_{3} \cos (\pi \alpha / 2) \omega^{\alpha}+e_{4}, \quad p_{2}(\omega)=\omega^{4 \alpha} \sin (2 \pi \alpha)+e_{1}$ $\sin (3 \pi \alpha / 2) \omega^{3 \alpha}+e_{2} \omega^{2 \alpha} \sin (\pi \alpha)+e_{3} \sin (\pi \alpha / 2) \omega^{\alpha}, q_{1}(\omega)=$ $f_{1} \omega^{2 \alpha} \cos (\pi \alpha)+f_{2} \cos (\pi \alpha / 2) \omega^{\alpha}+f_{3}$, and $q_{2}(\omega)=f_{1} \omega^{2 \alpha}$ $\sin (\pi \alpha)+f_{2} \sin (\pi \alpha / 2) \omega^{\alpha}$.

By $\cos ^{2}(\theta)+\sin ^{2}(\theta)=1$, then one has

$$
H_{1}(\omega)^{2}+H_{2}(\omega)^{2}=1
$$

Without loss of generality, we further suppose that Equation (16) has at least a positive root. By following from Equation (15), we can obtain

$$
\tau_{k}^{1}=\frac{1}{\omega}\left(\arccos \frac{-p_{1}(\omega) q_{1}(\omega)-p_{2}(\omega) q_{2}(\omega)}{p_{1}^{2}(\omega)+p_{2}^{2}(\omega)}+2 \pi\right), k=0,1,2, \cdots
$$

For the convenience of further analysis, we define the bifurcation point

$$
\tau_{1}^{0}=\min \left\{\tau_{k}^{1}\right\},(k=0,1,2 \cdots,) .
$$

By differentiating Equation (10) with respect to $\tau$, we have 


$$
\begin{gathered}
P^{\prime}(s) \frac{d s}{d \tau}+Q^{\prime}(s) \frac{d s}{d \tau} e^{-s \tau}+Q(s)\left(-\tau \frac{d s}{d \tau}-s\right) e^{-s \tau}=0, \\
\frac{d s}{d \tau}=\frac{s Q(s) e^{-s \tau}}{P^{\prime}(s)+\left(Q^{\prime}(s)-\tau Q(s)\right) e^{-s \tau}}=\frac{A(s)}{B(s)},
\end{gathered}
$$

and it implies

$$
\left.\operatorname{Re}\left[\frac{d s}{d \tau}\right]\right|_{\omega=\omega_{0}, \tau=\tau_{1}^{0}}=\frac{A_{1} B_{1}+A_{2} B_{2}}{B_{1}^{2}+B_{2}^{2}}
$$

The detailed calculations can be found in Appendix A.

Theorem 4. Supposing $\left(\left(A_{1} B_{1}+A_{2} B_{2}\right) /\left(B_{1}^{2}+B_{2}^{2}\right)\right) \neq 0$. For system (3), the following results can be obtained.

(i) If (H1), then the equilibrium $E(0,0,0,0)$ is locally asymptotically stable when $\tau \in\left[0, \tau_{1}^{0}\right)$

(ii) System (3) undergoes a Hopf bifurcation at $E(0,0,0$ ,0) when $\tau=\tau_{1}^{0}$

Case 2. Stability of $E_{1}\left(x_{1}^{*}, 0, x_{3}^{*}, 0\right)$ with $\tau_{1}=\tau_{2}=\tau$.

Let $X_{1}=x_{1}-x_{1}^{*}, X_{2}=x_{2}, X_{3}=x_{3}-x_{3}^{*}$, and $X_{4}=x_{4}$, then we can obtain linearized system:

$$
\left\{\begin{array}{l}
D^{\alpha} X_{1}=X_{2}, \\
D^{\alpha} X_{2}=n_{1} X_{1}-b_{1} X_{2}+a_{1} X_{3}+d_{1} X_{4}, \\
D^{\alpha} X_{3}=X_{4}, \\
D^{\alpha} X_{4}=a_{2} X_{1}+d_{2} X_{2}-\omega_{2}^{2} X_{3}-b_{2} X_{4}+n_{2} X_{3}(t-\tau),
\end{array}\right.
$$

where $n_{1}=-\omega_{1}^{2}+2 l_{1} x_{1}^{*}+3 l_{2}\left(x_{1}^{*}\right)^{2}$ and $n_{2}=\beta_{1}+3\left(x_{3}^{*}\right)^{2} \beta_{2}$.

The characteristic system (21) becomes

$$
s^{4 \alpha}+\varepsilon_{1} s^{3 \alpha}+\varepsilon_{2} s^{2 \alpha}+\varepsilon_{3} s^{\alpha}+\varepsilon_{4}+\left(\zeta_{1} s^{2 \alpha}+\zeta_{2} s^{\alpha}+\zeta_{3}\right) \mathrm{e}^{-\tau s}=0,
$$

where $\varepsilon_{1}=b_{1}+b_{2}, \quad \varepsilon_{2}=b_{1} b_{2}-n_{1}+\omega_{2}^{2}-d_{1} d_{2}, \quad \varepsilon_{3}=-b_{2}$ $n_{1}+b_{1} \omega_{2}^{2}-d_{1} a_{2}-d_{2} a_{1}, \varepsilon_{4}=-n_{1} \omega_{2}^{2}-a_{1} a_{2}, \zeta_{1}=-n_{2}, \zeta_{2}=-$ $b_{1} n_{2}$, and $\zeta_{3}=n_{2} n_{1}$.

Remark 5. Since Case 2 is quite similar to those for Case 1, we put the detailed mathematical derivation in Appendix B. Here, we only give the corresponding conclusions.

Theorem 6. Supposing $\left(\left(C_{1} D_{1}+C_{2} D_{2}\right) /\left(D_{1}^{2}+D_{2}^{2}\right)\right) \neq 0$. For system (3), the following results can be obtained.

(i) The equilibrium point $E_{1}\left(x_{1}^{*}, 0, x_{3}^{*}, 0\right)$ is locally asymptotically stable when $\tau \in\left(0, \tau^{0}\right)$ (ii) System (3) undergoes a Hopf bifurcation at $E_{1}\left(x_{1}^{*}\right.$, $\left.0, x_{3}^{*}, 0\right)$ when $\tau=\tau^{0}$

\section{Numerical Simulation}

In this section, we use the Adama-Bashforth-Moulton predictor-corrector method [31, 32] and show some numerical simulations to illustrate the analysis results in the previous sections. By choosing $\tau_{1}$ or $\tau_{2}$ as bifurcation parameters, we mainly analyse the Hopf bifurcation of system (3).

Case 1. To facilitate numerical simulations, the parameters take the values based on Ref. [16], as follows:

$$
a_{1}=6, a_{2}=4.5, b_{1}=2, b_{2}=0.5, d_{1}=1, d_{2}=0.5, \omega_{1}^{2}=7,
$$
$\omega_{2}^{2}=5, l_{1}=0.5, l_{2}=-0.1, \beta_{1}=0.8$ and $\beta_{2}=0.4$.

By performing some calculations based on previous analysis, we can obtain $\tau_{1}^{0}=5.1257$. Figure 1 shows that bifurcation diagrams of system (3) for the parameters $a_{2} \epsilon$ (2,4.5) with $\alpha=0.95, \tau_{1}=0$, and $h=2^{-6}$. The above parameters satisfy the conditions of Theorem 4; then, it demonstrates that $E_{0}(0,0,0,0)$ is locally asymptotically stable when $\tau_{1}=4.8<\tau_{1}^{0}$ and fractional-order $\alpha=0.95$, as shown in Figures 2 and 3. Besides, as $\tau_{1}$ increases, the Hopf bifurcation will occur at the expense of system's (3) stability. Figures 4 and 5 show that the solution of system (3) is unstable when $\tau_{1}=5.13>\tau_{1}^{0}$. The phase diagram depicts that the system undergoes a stable limit cycle around zero equilibrium point $E_{0}(0,0,0,0)$, as shown in Figure 5.

When $\tau_{1}=1$, although the system (3) is asymptotically stable with different values of the fractional order, the time required for the system to reach the stable state becomes long as the fractional-order value decreases, as shown in Figure 6 (can be found in Appendix C). It demonstrates that not only the time delay has an impact on the stability of the system, but the fractional order also plays an important role on the system stability.

Case 2. Fixed $\alpha=0.98, a_{1}=6, a_{2}=5, b_{1}=2, b_{2}=0.5, d_{1}=1$, $d_{2}=0.5, \omega_{1}^{2}=7, \omega_{2}^{2}=5, l_{1}=0.5, l_{2}=-0.1, \beta_{1}=1$ and $\beta_{2}=-$ 0.04 . By some calculations, we can obtain $x_{1}^{*}=3.6196, x_{3}^{*}$ $=3.9215$, and $\tau^{0}=1.0750$. Furthermore, we can get nonzero equilibrium $E_{1}(3.6196,0,3.9215,0)$ of the system (3). The above parameters satisfy the conditions of Theorem 6; then, it demonstrate that $E_{1}(3.6196,0,3.9215,0)$ is locally asymptotically stable when $\tau=1<\tau^{0}$ and fractional-order $\alpha=$ 0.98, as shown in Figures 7 and 8. Besides, as $\tau$ increases, the Hopf bifurcation will occur at the expense of system's (3) stability. Figures 9 and 10 show that the solution of system (3) is unstable when $\tau=1.2>\tau^{0}$. The phase diagram depicts that the system undergoes a stable limit cycle around nonzero equilibrium point $E_{1}(3.6196,0,3.9215,0)$, as shown in Figure 10.

When the time delay is small $(\tau=1)$, the stability of the system can remain stable as the value of the fractional order gradually increases. However, when the time delay becomes 


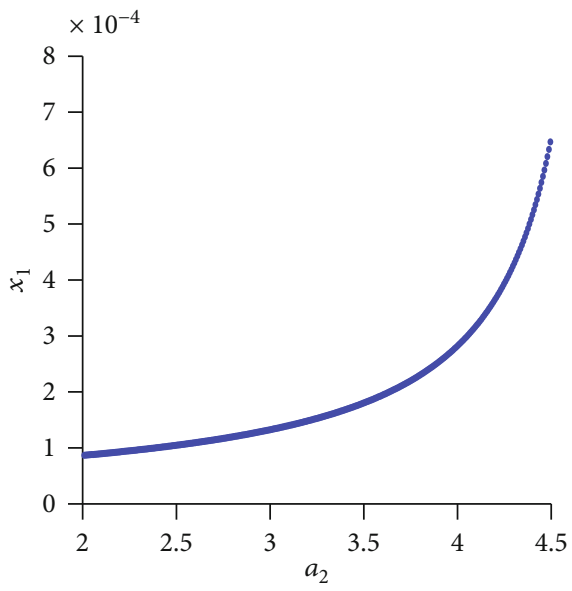

(a)

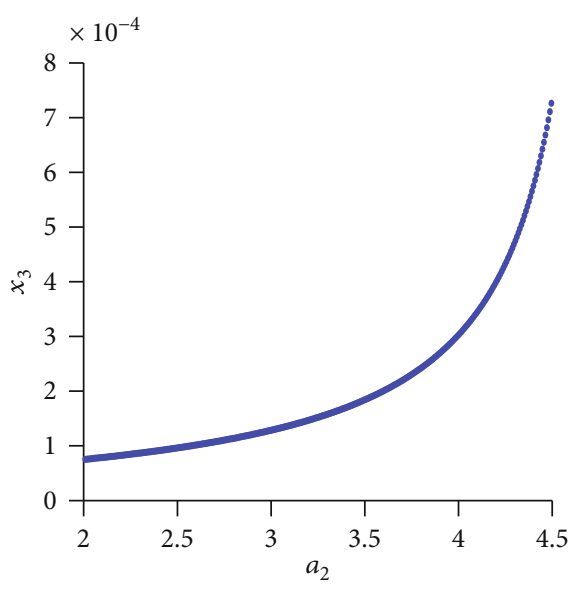

(c)

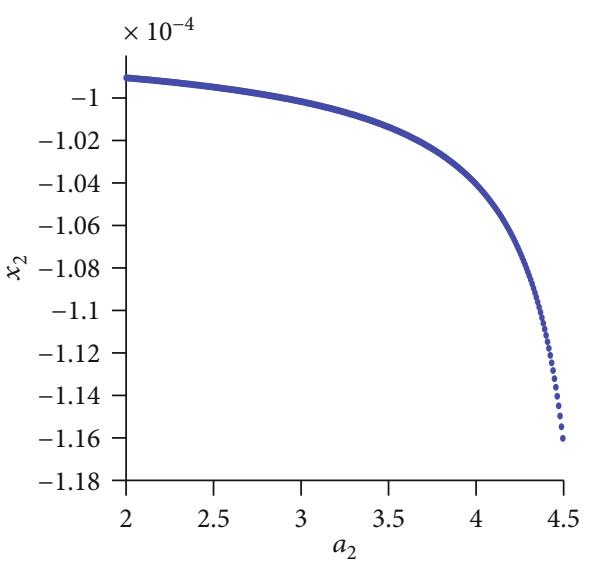

(b)

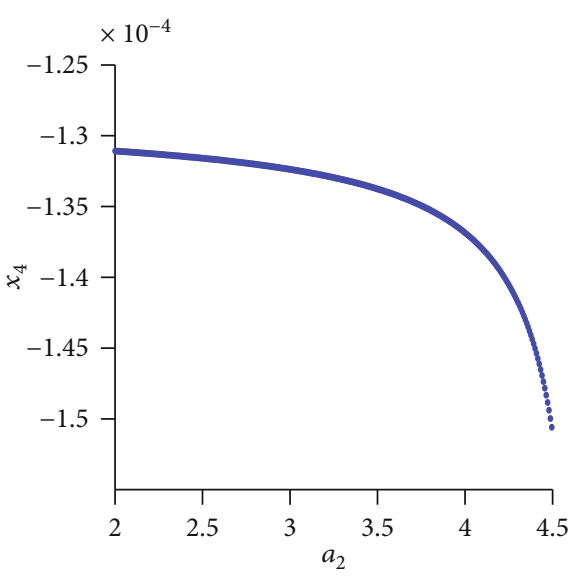

(d)

FIgURE 1: Bifurcation diagrams of system (3) for the parameters $a_{2} \in(2,4.5)$ with $\alpha=0.95$ and $h=2^{-6}$.

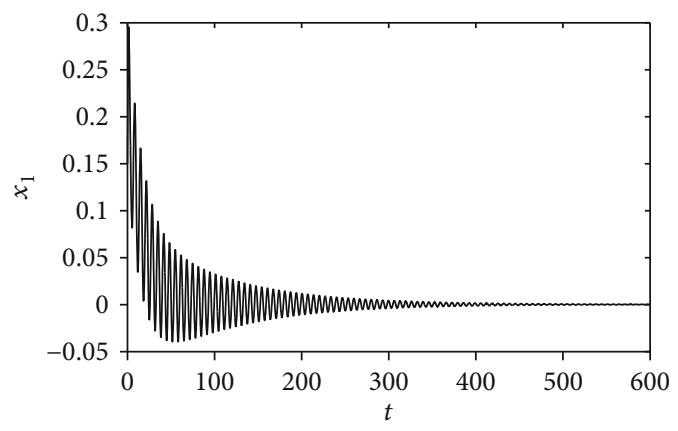

Figure 2: The system (3) at $E_{0}(0,0,0,0)$ is locally asymptotically stable, with $\alpha=0.95, \tau_{1}=4.8<\tau_{1}^{0}, h=0.05$, and $\left(x_{1}^{0}, x_{2}^{0}, x_{3}^{0}, x_{4}^{0}\right)=$ $(0.15,0.15,0.2,0.2)$.

big $(\tau=4)$, the nonzero equilibrium point of the system (3) changes from stable to unstable as the value of fractional order increases; finally, a periodic solution is generated, as shown in Figure 11 (can be found in Appendix C). It shows that the fractional order also plays an important role on the stability of the system.

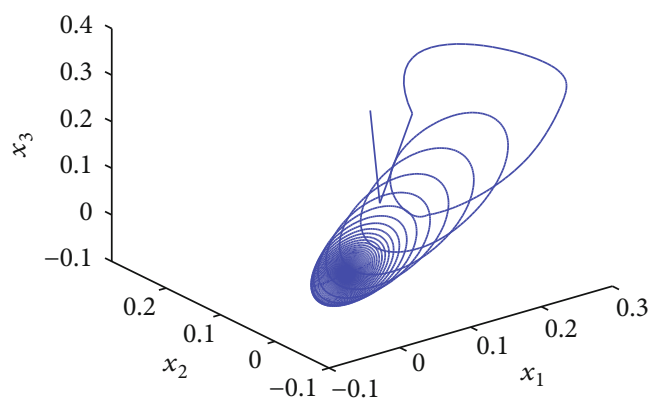

Figure 3: Phase portraits of system (3) at $E_{0}(0,0,0,0)$ with $\alpha=0.95$, $\tau_{1}=4.8<\tau_{1}^{0}, h=0.05$, and $\left(x_{1}^{0}, x_{2}^{0}, x_{3}^{0}, x_{4}^{0}\right)=(0.15,0.15,0.2,0.2)$.

\section{Discussions}

In Refs. [22-25], when introducing fractional orders to existing systems, the usual method is to directly replace the integer order with the fractional order. Accordingly, we follow the same processing method to introduce the fractional order into system (2). However, such an approach may ignore the relevant mechanics [33] of the actual system. The coupled systems [33] and damping models [34-36] 


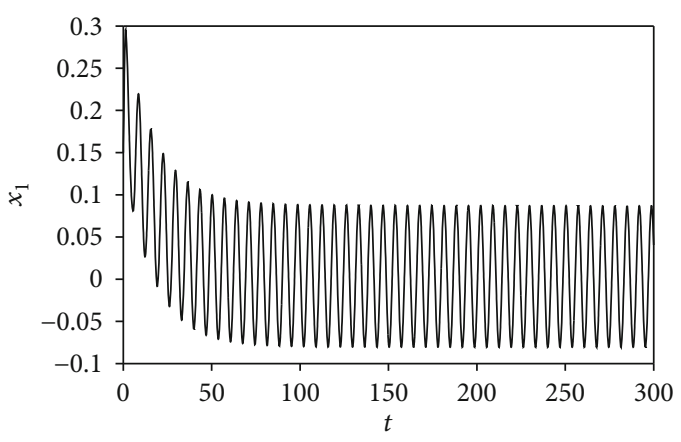

FIgure 4: The system (3) at $E_{0}(0,0,0,0)$ is unstable, with $\alpha=0.95$, $\tau_{1}=5.13>\tau_{1}^{0}, h=0.05$, and $\left(x_{1}^{0}, x_{2}^{0}, x_{3}^{0}, x_{4}^{0}\right)=(0.15,0.15,0.2,0.2)$.

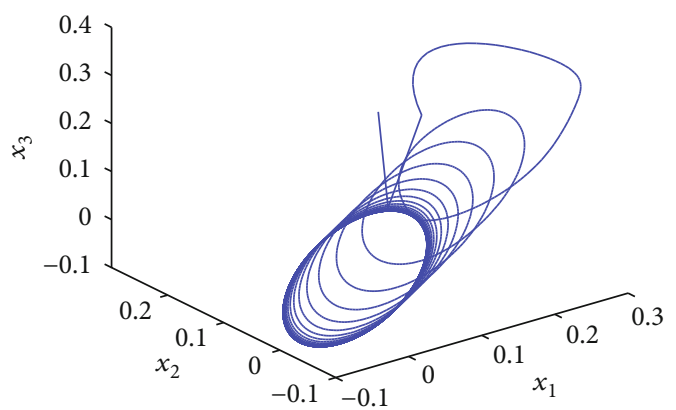

Figure 5: Phase portraits of system (3) at $E_{0}(0,0,0,0)$ with $\alpha=0.95$, $\tau_{1}=5.13>\tau_{1}^{0}, h=0.05$, and $\left(x_{1}^{0}, x_{2}^{0}, x_{3}^{0}, x_{4}^{0}\right)=(0.15,0.15,0.2,0.2)$.

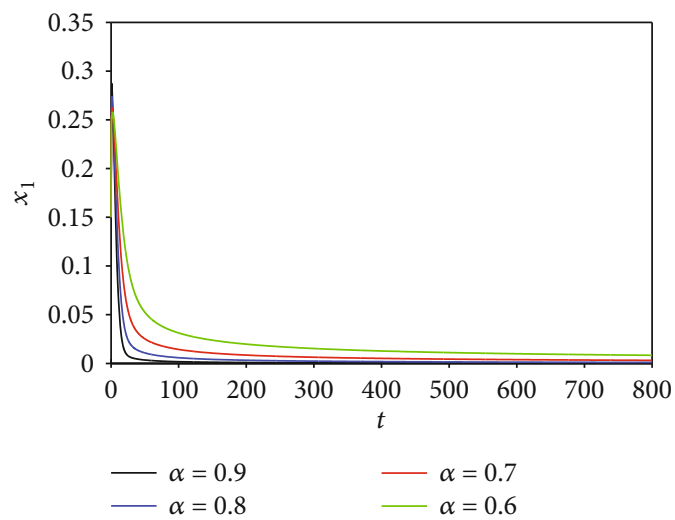

Figure 6: Waveform diagrams of system (3) at $E_{0}(0,0,0,0)$ with $\tau=1, h=0.05$, and $\left(x_{1}^{0}, x_{2}^{0}, x_{3}^{0}, x_{4}^{0}\right)=(0.15,0.15,0.2,0.2)$.

using fractional derivatives had been successfully applied to many dynamic systems in mechanical engineering. Inspired by the above literatures, we introduce fractional order in the coupling term $C\left(\dot{\varphi}_{1}-\dot{\varphi}_{2}\right)$ and the damping term $C_{\mathrm{e}} \dot{\varphi}_{1}$ in system (2); then, we have

$\left\{\begin{array}{c}J_{1} \ddot{\varphi}_{1}+K\left(\varphi_{1}-\varphi_{2}\right)+C D^{\alpha} \varphi_{1}-C^{\prime} D^{\beta} \varphi_{2}+C_{e} D^{\alpha} \varphi_{1}-K_{e} \varphi_{1}=k_{0}+k_{2} \varphi_{1}^{2}+k_{3} \varphi_{1}^{3}, \\ J_{2} \ddot{\varphi}_{2}-K\left(\varphi_{1}-\varphi_{2}\right)-C D^{\alpha} \varphi_{1}+C^{\prime} D^{\beta} \varphi_{2}=T_{L}+g_{c 1} \varphi_{2}\left(t-\tau_{1}\right)+g_{c 2} \varphi_{2}^{3}\left(t-\tau_{2}\right),\end{array}\right.$

where $\alpha, \beta \in(0,1], \alpha \neq \beta, D^{\alpha}$, and $D^{\beta}$ denote the GrünwaldLetnikov fractional derivative.

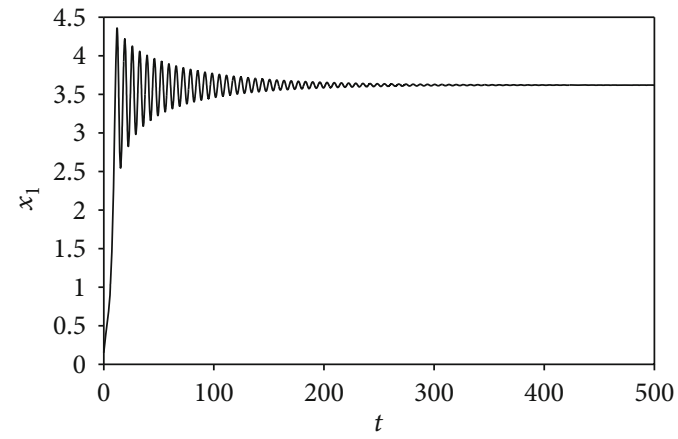

FIgURE 7: The system (3) at $E_{1}(3.6196,0,3.9215,0)$ is locally asymptotically stable, with $\alpha=0.98, \tau=1<\tau^{0}, h=0.05$, and $\left(x_{1}^{0}\right.$, $\left.x_{2}^{0}, x_{3}^{0}, x_{4}^{0}\right)=(0.15,0.15,0.2,0.2)$.

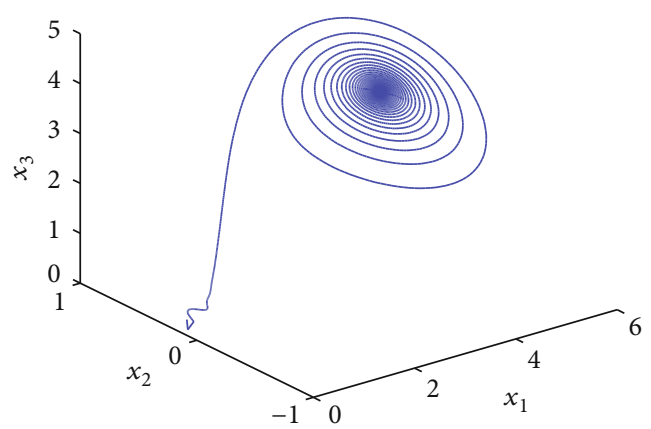

Figure 8: Phase portraits of system (3) at $E_{1}(3.6196,0,3.9215,0)$ with $\quad \alpha=0.98, \quad \tau=1<\tau^{0}, \quad h=0.05, \quad$ and $\quad\left(x_{1}^{0}, x_{2}^{0}, x_{3}^{0}, x_{4}^{0}\right)=($ $0.15,0.15,0.2,0.2)$.

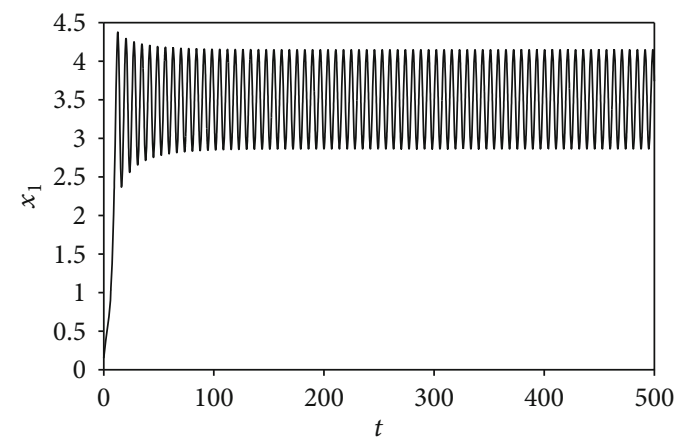

Figure 9: The system (3) at $E_{1}(3.6196,0,3.9215,0)$ has a stable periodic solution, with $\alpha=0.98, \tau=1.2>\tau^{0}, h=0.05$, and $\left(x_{1}^{0}, x_{2}^{0}\right.$, $\left.x_{3}^{0}, x_{4}^{0}\right)=(0.15,0.15,0.2,0.2)$.

Based on the additivity of the fractional-order derivative operator, we reduce the dimensionality of Equation (23), and Equation (23) then becomes

$\left\{\begin{array}{l}D^{\alpha} \varphi_{1}=\varphi_{3}, \\ D^{2-\alpha} \varphi_{3}=\ddot{\varphi}_{1}=\frac{1}{J_{1}}\left(-K\left(\varphi_{1}-\varphi_{2}\right)-C \varphi_{3}+C^{\prime} \varphi_{4}-C_{e} \varphi_{3}+K_{e} \varphi_{1}+k_{0}+k_{2} \varphi_{1}^{2}+k_{3} \varphi_{1}^{3}\right), \\ D^{\beta} \varphi_{2}=\varphi_{4}, \\ D^{2-\beta} \varphi_{4}=\ddot{\varphi}_{2}=\frac{1}{J_{2}}\left(K\left(\varphi_{1}-\varphi_{2}\right)+C \varphi_{3}-C^{\prime} \varphi_{4}+T_{L}+g_{c 1} \varphi_{2}\left(t-\tau_{1}\right)+g_{c 2} \varphi_{2}^{3}\left(t-\tau_{2}\right)\right) .\end{array}\right.$ 


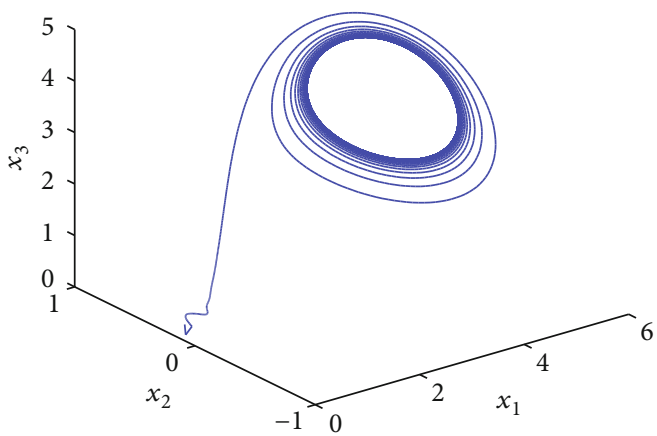

Figure 10: Phase portraits of system (3) at $E_{1}(3.6196,0,3.9215,0)$ with $\alpha=0.98, \tau=1.2>\tau^{0}, h=0.05$, and $\left(x_{1}^{0}, x_{2}^{0}, x_{3}^{0}, x_{4}^{0}\right)=($ $0.15,0.15,0.2,0.2)$.

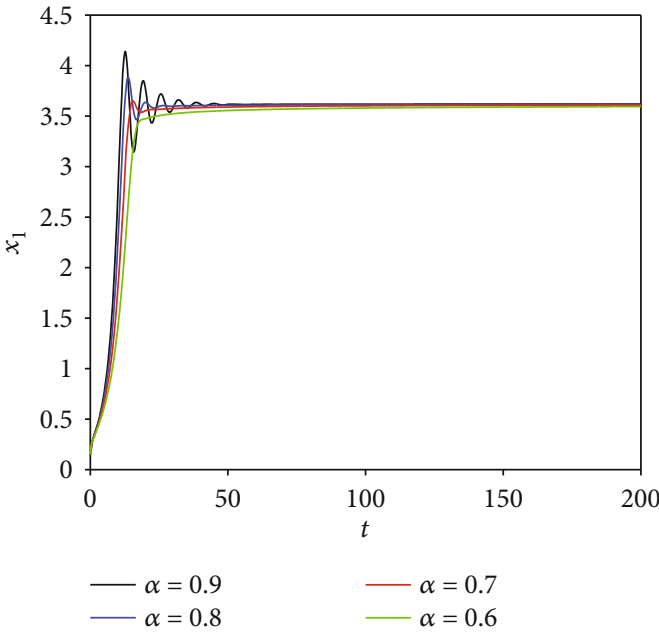

(a)

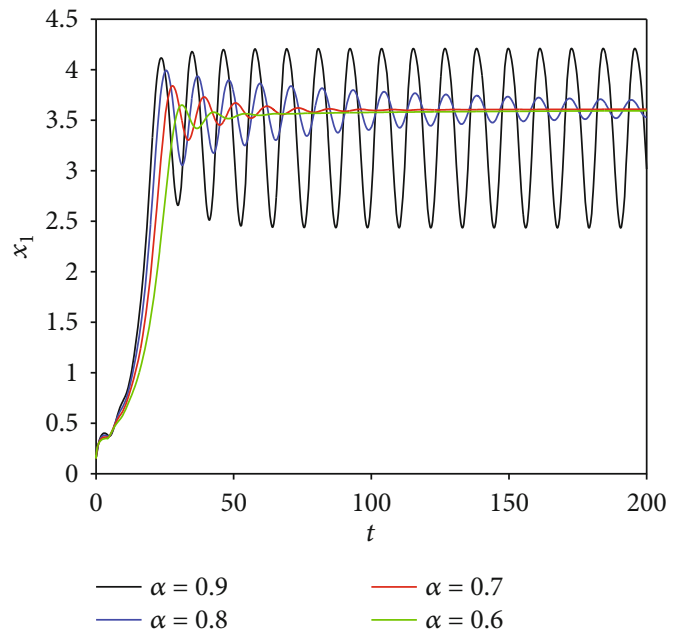

(b)

FIGURE 11: Waveform diagrams of system (3) at $E_{1}(3.6196,0,3.9215,0)$ with $h=0.05$ and $\left(x_{1}^{0}, x_{2}^{0}, x_{3}^{0}, x_{4}^{0}\right)=(0.15,0.15,0.2,0.2)$. (a) $\tau=1$; (b) $\tau=4$.

Let $\alpha_{1}=\alpha, \alpha_{2}=2-\alpha, \alpha_{3}=\beta, \alpha_{4}=2-\beta, \varphi_{1}=x_{1}, \varphi_{3}=x_{2}$, $\varphi_{2}=x_{3}, \varphi_{4}=x_{4}, \omega_{1}^{2}=\left(K-K_{\mathrm{e}}\right) / J_{1} b_{1}=\left(C+C_{\mathrm{e}}\right) / J_{1}, \quad a_{1}=K /$ $J_{1}, d_{1}^{\prime}=C^{\prime} / J_{1}, l_{1}=k_{2} / J_{1}, l_{2}=k_{3} / J_{1}, \omega_{2}^{2}=K / J_{2}, b_{2}^{\prime}=C^{\prime} / J_{2}, a_{2}=$ $K / J_{2}, d_{2}=C / J_{2}, \beta_{1}=g_{\mathrm{cl}} / J_{2}, \beta_{2}=g_{\mathrm{c} 2} / J_{2}$, then Equation (13) can be rewritten as

$$
\left\{\begin{array}{l}
D^{\alpha_{1}} x_{1}=x_{2}, \\
D^{\alpha_{2}} x_{2}=-\omega_{1}^{2} x_{1}-b_{1} x_{2}+a_{1} x_{3}+d_{1}^{\prime} x_{4}+l_{1} x_{1}^{2}+l_{2} x_{1}^{3}, \\
D^{\alpha_{3}} x_{3}=x_{4}, \\
D^{\alpha_{4}} x_{4}=a_{2} x_{1}+d_{2} x_{2}-\omega_{2}^{2} x_{3}-b_{2}^{\prime} x_{4}+\beta_{1} x_{3}\left(t-\tau_{1}\right)+\beta_{2} x_{3}^{3}\left(t-\tau_{2}\right),
\end{array}\right.
$$

where $\alpha_{i}(i=1,3) \in(0,1], \alpha_{i}(i=2,4) \in[1,2)$, and $D^{\alpha_{i}}(i=1$, $2,3,4)$ denotes the Grünwald-Letnikov fractional derivative. It is clear to find that system (25) can be seen as a more general form of system (3), which better fits the actual model. Therefore, further analysis of the dynamics of system (25) will be our future work.

\section{Conclusions}

Bifurcation analysis can answer how do the behavior of the system change as the parameters change. In this work, we propose a fractional-order nonlinear electromechanical coupling system with delay. By choosing time delay $\tau$ as a bifurcation parameter, we, respectively, derive the conditions for occurrence of Hopf bifurcation around zero equilibrium $E_{0}$ $(0,0,0,0)$ and nonzero equilibrium $E_{1}\left(x_{1}^{*}, 0, x_{3}^{*}, 0\right)$. It is demonstrated that fractional-order $\alpha$ and time delay $\tau$ have an important influence on electromechanical coupling system. By applying Adams-Bashforth-Moulton method, we implement some simulations to corroborate our analysis results.

For electromechanical coupling system, the unstable bifurcation may cause a destructive vibration. Our analysis results demonstrate that a combination of fractional order $\alpha$ and time delay $\tau$ in the system have an important effect on the dynamic properties of the system. From the results of the numerical simulation, selecting the fractional order $\alpha$ and $a_{2}$ and the feedback parameters can realize the switching of the dynamic properties of the system from a zero equilibrium point to a nonzero equilibrium point. Here, $a_{2}$ 
represents the ratio of the torsional stiffness of drive shaft $K$ to the moment of inertia $J_{2}$ (see [16]). Thus, our obtained results may have some implications for reducing the vibration of electromechanical coupled rolling mill systems.

Besides, it was found that the fractal derivative is the mathematical approximation of the fractional derivative in engineering applications, and the fractal derivative can describe the randomness of nonlinear systems (see [37-41]). Therefore, fractional-order systems may be the basis for many future studies. It will be our future work to study the randomness of rolling mill vibration by using fractional-order (or fractal derivative) mathematical modeling.

\section{Appendix}

\section{A. The Expression of Parameters in Equation} (8) and Equation (20)

$$
\begin{aligned}
& m_{1}=-\frac{l_{2}^{3} \beta_{2}}{a_{1}^{3}}, m_{2}=-\frac{3 l_{1} l_{2}^{2} \beta_{2}}{a_{1}^{3}}, m_{3} \\
& =\frac{\beta_{2}}{a_{1}^{3}}\left(l_{2}^{2} \omega_{1}^{2}-2 l_{1}^{2} l_{2}+2 l_{2}^{2} \omega_{1}^{2}-2 l_{1}^{2} l_{2}\right), m_{4} \\
& =\frac{\beta_{2}}{a_{1}^{3}}\left(2 l_{1} l_{2} \omega_{1}^{2}-2 l_{1}^{3}+4 l_{1} l_{2} \omega_{1}^{2}\right), m_{5} \\
& \left.=\frac{\beta_{2}}{a_{1}^{3}}\left(2 l_{1} \omega_{1}^{2}-\omega_{1}^{2}\left(2 l_{2} \omega_{1}^{2}-l_{1}^{2}\right)-l_{2} \omega_{1}^{4}\right)\right), m_{6} \\
& =-\frac{l_{1} \omega_{1}^{4} \beta_{2}}{a_{1}^{3}}, m_{7}=\frac{\omega_{1}^{6} \beta_{2}}{a_{1}^{3}}+\frac{l_{2}\left(\omega_{2}^{2}-\beta_{1}\right)}{a_{1}}, m_{8} \\
& =\frac{l_{1}\left(\omega_{2}^{2}-\beta_{1}\right)}{a_{1}}, m_{9}=-\frac{\omega_{1}^{2}\left(\omega_{2}^{2}-\beta_{1}\right)}{a_{1}}+a_{2} \text {. } \\
& A_{1}=\omega_{0}\left[\left(f_{1} \omega^{2 \alpha} \cos (\alpha \pi)+f_{2} \omega^{\alpha} \cos \left(\frac{\alpha \pi}{2}\right)+f_{3}\right) \sin \left(\omega_{0} \tau_{1}^{0}\right)\right. \\
& \left.-\left(f_{1} \omega^{2 \alpha} \sin (\alpha \pi)+f_{2} \omega^{\alpha} \sin \left(\frac{\alpha \pi}{2}\right)\right) \cos \left(\omega_{0} \tau_{1}^{0}\right)\right], \\
& A_{2}=\omega_{0}\left[\left(f_{1} \omega^{2 \alpha} \cos (\alpha \pi)+f_{2} \omega^{\alpha} \cos \left(\frac{\alpha \pi}{2}\right)+f_{3}\right) \cos \left(\omega_{0} \tau_{1}^{0}\right)\right. \\
& \left.+\left(f_{1} \omega^{2 \alpha} \sin (\alpha \pi)+f_{2} \omega^{\alpha} \sin \left(\frac{\alpha \pi}{2}\right)\right) \sin \left(\omega_{0} \tau_{1}^{0}\right)\right], \\
& B_{1}=4 \alpha \omega^{4 \alpha-1} \cos \left(\frac{(4 \alpha-1) \pi}{2}\right)+3 \alpha e_{1} \omega^{3 \alpha-1} \cos \left(\frac{(3 \alpha-1) \pi}{2}\right) \\
& +2 \alpha e_{2} \omega^{2 \alpha-1} \cos \left(\frac{(2 \alpha-1) \pi}{2}\right)+\alpha e_{3} \omega^{\alpha-1} \cos \left(\frac{(\alpha-1) \pi}{2}\right) \\
& +\cos \left(\omega_{0} \tau_{1}^{0}\right)\left(2 \alpha f_{1} \omega^{2 \alpha-1} \cos \left(\frac{(2 \alpha-1) \pi}{2}\right)\right. \\
& +\alpha f_{2} \omega^{\alpha-1} \cos \left(\frac{(\alpha-1) \pi}{2}\right)-\tau_{1}^{0} f_{1} \omega^{2 \alpha} \cos \left(\frac{2 \alpha \pi}{2}\right) \\
& \left.-\tau_{1}^{0} f_{2} \omega^{\alpha} \cos \left(\frac{\alpha \pi}{2}\right)-\tau_{1}^{0} f_{3}\right)-\sin \left(\omega_{0} \tau_{1}^{0}\right) \\
& \cdot\left(2 \alpha f_{1} \omega^{2 \alpha-1} \sin \left(\frac{(2 \alpha-1) \pi}{2}\right)+\alpha f_{2} \omega^{\alpha-1} \sin \left(\frac{(\alpha-1) \pi}{2}\right)\right. \\
& -\tau_{1}^{0} f_{1} \omega^{2 \alpha} \sin \left(\frac{2 \alpha \pi}{2}\right)-\tau_{1}^{0} f_{2} \omega^{\alpha} \sin \left(\frac{\alpha \pi}{2}\right)
\end{aligned}
$$

$$
\begin{aligned}
B_{2}= & 4 \alpha \omega^{4 \alpha-1} \sin \left(\frac{(4 \alpha-1) \pi}{2}\right)+3 \alpha e_{1} \omega^{3 \alpha-1} \sin \left(\frac{(3 \alpha-1) \pi}{2}\right) \\
& +2 \alpha e_{2} \omega^{2 \alpha-1} \sin \left(\frac{(2 \alpha-1) \pi}{2}\right)+\alpha e_{3} \omega^{\alpha-1} \sin \left(\frac{(\alpha-1) \pi}{2}\right) \\
& +\sin \omega_{0} \tau_{1}^{0}\left(2 \alpha f_{1} \omega^{2 \alpha-1} \cos \frac{(2 \alpha-1) \pi}{2}\right. \\
& -\alpha f_{2} \omega^{\alpha-1} \cos \left(\frac{(\alpha-1) \pi}{2}\right)-\tau_{1}^{0} f_{1} \omega^{2 \alpha} \cos \left(\frac{2 \alpha \pi}{2}\right) \\
& \left.-\tau_{1}^{0} f_{2} \omega^{\alpha} \cos \left(\frac{\alpha \pi}{2}\right)-\tau_{0} f_{3}\right)+\cos \left(\omega_{0} \tau_{1}^{0}\right) \\
& \cdot\left(2 \alpha f_{1} \omega^{2 \alpha-1} \sin \left(\frac{(2 \alpha-1) \pi}{2}\right)+\alpha f_{2} \omega^{\alpha-1} \sin \left(\frac{(\alpha-1) \pi}{2}\right)\right. \\
& -\tau_{1}^{0} f_{1} \omega^{2 \alpha} \sin \left(\frac{2 \alpha \pi}{2}\right)-\tau_{1}^{0} f_{2} \omega^{\alpha} \sin \left(\frac{\alpha \pi}{2}\right) .
\end{aligned}
$$

\section{B. Theoretical Derivation of Stability and Hopf Bifurcation of System (21)}

For the convenience of further analysis, we denote $\Phi(s)=$ $s^{4 \alpha}+\varepsilon_{1} s^{3 \alpha}+\varepsilon_{2} s^{2 \alpha}+\varepsilon_{3} s^{\alpha}+\varepsilon_{4}$ and $\Psi(s)=\zeta_{1} s^{2 \alpha}+\zeta_{2} s^{\alpha}+\zeta_{3}$. Then, Equation (22) is equivalent to

$$
\Phi(s)+e^{-\tau s} \Psi(s)=0 .
$$

Multiplying $e^{s \tau}$ on both sides of Equation (B.1), then we get

$$
\Phi(s) e^{\tau s}+\Psi(s)=0
$$

By replacing $s=i \omega=(\cos (\pi / 2)+i \sin (\pi / 2)) \omega$ into Equation (B.2) and separating into its real and imaginary parts, we can obtain

$$
\begin{gathered}
\left(\phi_{1}(\omega)+i \phi_{2}(\omega)\right)(\cos (\omega \tau)+i \sin (\omega \tau))+\psi_{1}(\omega)+i \psi_{2}(\omega)=0, \\
\left\{\begin{array}{l}
\phi_{1}(\omega) \cos (\omega \tau)-\phi_{2}(\omega) \sin (\omega \tau)+\psi_{1}(\omega)=0, \\
\phi_{2}(\omega) \cos (\omega \tau)+\phi_{1}(\omega) \sin (\omega \tau)+\psi_{2}(\omega)=0
\end{array}\right.
\end{gathered}
$$

where $\Phi(i \omega)=\phi_{1}(\omega)+i \phi_{2}(\omega)$ and $\Psi(i \omega)=\psi_{1}(\omega)+i \psi_{2}(\omega)$. Then, by solving Equation (B.3), one obtains

$$
\left\{\begin{array}{l}
\cos (\omega \tau)=\frac{-\phi_{1}(\omega) \psi_{1}(\omega)-\phi_{2}(\omega) \psi_{2}(\omega)}{\phi_{1}^{2}(\omega)+\phi_{2}^{2}(\omega)} \doteq Y_{1}(\omega), \\
\sin (\omega \tau)=\frac{-\phi_{1}(\omega) \psi_{2}(\omega)+\phi_{2}(\omega) \psi_{1}(\omega)}{\phi_{1}^{2}(\omega)+\phi_{2}^{2}(\omega)} \doteq Y_{2}(\omega),
\end{array}\right.
$$

where $\quad \phi_{1}(\omega)=\omega^{4 \alpha} \cos (2 \pi \alpha)+\varepsilon_{1} \cos (3 \pi \alpha / 2) \omega^{3 \alpha}+\varepsilon_{2} \omega^{2 \alpha}$ $\cos (\pi \alpha)+\varepsilon_{3} \cos (\pi \alpha / 2) \omega^{\alpha}+\varepsilon_{4}, \quad \phi_{2}(\omega)=\omega^{4 \alpha} \sin (2 \pi \alpha)+\varepsilon_{1}$ $\sin (3 \pi \alpha / 2) \omega^{3 \alpha}+\varepsilon_{2} \omega^{2 \alpha} \sin (\pi \alpha)+\varepsilon_{3} \sin \left((\pi \alpha / 2) \omega^{\alpha}\right), \psi_{1}(\omega)$ 
$=\zeta_{1} \omega^{2 \alpha} \cos (\pi \alpha)+\zeta_{2} \cos (\pi \alpha / 2) \omega^{\alpha}+\zeta_{3}$, and $\psi_{2}(\omega)=\zeta_{1} \omega^{2 \alpha}$ $\sin (\pi \alpha)+\zeta_{2} \sin (\pi \alpha / 2) \omega^{\alpha}$.

From $\cos ^{2}(\theta)+\sin ^{2}(\theta)=1$, then

$$
Y_{1}(\omega)^{2}+Y_{2}(\omega)^{2}=1
$$

Without loss of generality, we further suppose that Equation (B.5) has at least a positive root. By following from Equation (B.4), we can obtain

$\tau_{k}=\frac{1}{\omega}\left(\arccos \frac{-\phi_{1}(\omega) \psi_{1}(\omega)-\phi_{2}(\omega) \psi_{2}(\omega)}{\phi_{1}^{2}(\omega)+\psi_{2}^{2}(\omega)}+2 \pi\right), k=0,1,2, \cdots$.

Furthermore, we define the bifurcation point $\tau^{0}=\min$ $\left\{\tau_{k}\right\}, k=0,1,2 \cdots$, have

By differentiating Equation (B.1) with respect to $\tau$, we

$$
\begin{gathered}
\Phi^{\prime}(s) \frac{d s}{d \tau}+\Psi^{\prime}(s) \frac{d s}{d \tau} e^{-s \tau}+\Psi(s)\left(-\tau \frac{d s}{d \tau}-s\right) e^{-s \tau}=0, \\
\frac{d s}{d \tau}=\frac{s \Psi(s) e^{-s \tau}}{\Phi^{\prime}(s)+\left(\Psi^{\prime}(s)-\tau \Psi(s)\right) e^{-s \tau}}=\frac{C(s)}{D(s)} .
\end{gathered}
$$

Then, it implies

$$
\left.\operatorname{Re}\left[\frac{d s}{d \tau}\right]\right|_{\omega=\omega_{0}, \tau=\tau^{0}}=\frac{C_{1} C_{1}+C_{2} D_{2}}{D_{1}^{2}+D_{2}^{2}},
$$

where

$$
\begin{aligned}
C_{1}= & \omega_{0}\left[\left(\zeta_{1} \omega^{2 \alpha} \cos (\alpha \pi)+\zeta_{2} \omega^{\alpha} \cos \left(\frac{\alpha \pi}{2}\right)+\zeta_{3}\right) \sin \left(\omega_{0} \tau^{0}\right)\right. \\
& \left.-\left(\zeta_{1} \omega^{2 \alpha} \sin (\alpha \pi)+\zeta_{2} \omega^{\alpha} \sin \left(\frac{\alpha \pi}{2}\right)\right) \cos \left(\omega_{0} \tau^{0}\right)\right], \\
C_{2}= & \omega_{0}\left[\left(\zeta_{1} \omega^{2 \alpha} \cos (\alpha \pi)+\zeta_{2} \omega^{\alpha} \cos \left(\frac{\alpha \pi}{2}\right)+\zeta_{3}\right) \cos \left(\omega_{0} \tau^{0}\right)\right. \\
& \left.+\left(\zeta_{1} \omega^{2 \alpha} \sin (\alpha \pi)+\zeta_{2} \omega^{\alpha} \sin \left(\frac{\alpha \pi}{2}\right)\right) \sin \left(\omega_{0} \tau^{0}\right)\right], \\
D_{1}= & 4 \alpha \omega^{4 \alpha-1} \cos \left(\frac{(4 \alpha-1) \pi}{2}\right)+3 \alpha \varepsilon_{1} \omega^{3 \alpha-1} \cos \left(\frac{(3 \alpha-1) \pi}{2}\right) \\
& +2 \alpha \varepsilon_{2} \omega^{2 \alpha-1} \cos \left(\frac{(2 \alpha-1) \pi}{2}\right)+\alpha \varepsilon_{3} \omega^{\alpha-1} \cos \left(\frac{(\alpha-1) \pi}{2}\right) \\
& +\cos \left(\omega_{0} \tau^{0}\right)\left(2 \alpha \zeta_{1} \omega^{2 \alpha-1} \cos \left(\frac{(2 \alpha-1) \pi}{2}\right)\right. \\
& +\alpha \zeta_{2} \omega^{\alpha-1} \cos \left(\frac{(\alpha-1) \pi}{2}\right)-\tau^{0} \zeta_{1} \omega^{2 \alpha} \cos \left(\frac{2 \alpha \pi}{2}\right) \\
& \left.-\tau^{0} \zeta_{2} \omega^{\alpha} \cos \left(\frac{\alpha \pi}{2}\right)-\tau^{0} \zeta_{3}\right)-\sin \left(\omega_{0} \tau^{0}\right) \\
& +\left(2 \alpha \zeta_{1} \omega^{2 \alpha-1} \sin \left(\frac{(2 \alpha-1) \pi}{2}\right)+\alpha \zeta_{2} \omega^{\alpha-1} \sin \left(\frac{(\alpha-1) \pi}{2}\right)\right. \\
& \left.-\tau^{0} \zeta_{1} \omega^{2 \alpha} \sin \left(\frac{2 \alpha \pi}{2}\right)-\tau^{0} \zeta_{2} \omega^{\alpha} \sin \left(\frac{\alpha \pi}{2}\right)\right),
\end{aligned}
$$

$$
\begin{aligned}
D_{2}= & 4 \alpha \omega^{4 \alpha-1} \sin \left(\frac{(4 \alpha-1) \pi}{2}\right)+3 \alpha \varepsilon_{1} \omega^{3 \alpha-1} \sin \left(\frac{(3 \alpha-1) \pi}{2}\right) \\
& +2 \alpha \varepsilon_{2} \omega^{2 \alpha-1} \sin \left(\frac{(2 \alpha-1) \pi}{2}\right)+\alpha \varepsilon_{3} \omega^{\alpha-1} \sin \left(\frac{(\alpha-1) \pi}{2}\right) \\
& +\sin \left(\omega_{0} \tau^{0}\right)\left(2 \alpha \zeta_{1} \omega^{2 \alpha-1} \cos \left(\frac{(2 \alpha-1) \pi}{2}\right)\right. \\
& -\alpha \zeta_{2} \omega^{\alpha-1} \cos \left(\frac{(\alpha-1) \pi}{2}\right)-\tau^{0} \zeta_{1} \omega^{2 \alpha} \cos \left(\frac{2 \alpha \pi}{2}\right) \\
& \left.-\tau^{0} \zeta_{2} \omega^{\alpha} \cos \left(\frac{\alpha \pi}{2}\right)-\tau^{0} \zeta_{3}\right)+\cos \left(\omega_{0} \tau^{0}\right) \\
& +\left(2 \alpha \zeta_{1} \omega^{2 \alpha-1} \sin \left(\frac{(2 \alpha-1) \pi}{2}\right)+\alpha \zeta_{2} \omega^{\alpha-1} \sin \left(\frac{(\alpha-1) \pi}{2}\right)\right. \\
& \left.-\tau^{0} \zeta_{1} \omega^{2 \alpha} \sin \left(\frac{2 \alpha \pi}{2}\right)-\tau^{0} \zeta_{2} \omega^{\alpha} \sin \left(\frac{\alpha \pi}{2}\right)\right) .
\end{aligned}
$$

C. Waveform Diagrams of System (3) at $E_{0}(0$, $0,0,0)$ and $E_{1}(3.6196,0,3.9215,0)$

\section{Data Availability}

The data used to support the findings of this study are included within the article.

\section{Conflicts of Interest}

The authors declare that they have no competing interests.

\section{Acknowledgments}

This work is supported by the Fund for Shanxi 1331 Project Key Subjects Construction.

\section{References}

[1] J. F. Wang and L. M. Cartright, “Torsional vibration modeling and dynamic simulation of a rolling stand power transmission system," Iron and Steel Engineer, vol. 76, no. 7, pp. 30-34, 1999.

[2] M. A. Younes, M. Shahtout, and M. N. Damir, "A parameters design approach to improve product quality and equipment performance in hot rolling," Journal of Materials Processing Technology, vol. 171, no. 1, pp. 83-92, 2006.

[3] Y. F. Zhang, X. Q. Yan, and Q. H. Ling, "Electromechanical coupling vibration of rolling mill excited by variable frequency harmonic," Advanced Materials Research, vol. 912-914, pp. 662-665, 2014.

[4] S. H. He and J. Zhong, "Modeling and identification of HAGC system of temper rolling mill," Journal of Central South University of Technology, vol. 12, no. 6, pp. 699-704, 2005.

[5] Y. Hori, H. Sawada, and Yeonghan Chun, "Slow resonance ratio control for vibration suppression and disturbance rejection in torsional system," IEEE Transactions on Industrial Electronics, vol. 46, no. 1, pp. 162-168, 1999.

[6] H. Jewik, R. P. Stratford, and C. W. Thomas, "Torque amplification and torsional vibration in large mill drives," IEEE Transactions on Industry and General Applications, vol. IGA5, no. 3, pp. 333-346, 1969. 
[7] J. J. He, S. Y. Yu, and J. Zhong, "Modeling for driving systems of four-high rolling mill," Transactions of Nonferrous Metals Society of China, vol. 12, no. 1, pp. 88-92, 2002.

[8] Z. Wang and D. Wang, "Method of judging the self-excited vibration of rolling main drive system in rolling slippage," Journal of Sound and Vibration, vol. 215, no. 5, pp. 11351143, 1998.

[9] W. Dobrucki and A. Bar, "Changes in roll-gap shape in the case of vibrations in a four-high rolling mill stand," Journal of Materials Processing Technology, vol. 61, no. 4, pp. 328337, 1996.

[10] G. Monaco, "Dynamics of rolling mills-mathematical models and experimental results," Iron and Steel Engineer, vol. 54, no. 12, pp. 35-46, 1977.

[11] B. Liu, S. Liu, Y. K. Zhang, and Y. Wen, "Bifurcation control for electromechanical coupling vibration in rolling mill drive system based on nonlinear feedback," Journal of Mechanical Engineering, vol. 8, 2010(in Chinese).

[12] R. C. Zhang and P. P. Yang, "Study on Hopf bifurcation of displacement delayed feedback system for main drive system of rolling mill," Machinery Design Manufacture, vol. 5, 2012, (in Chinese).

[13] S. Liu, S. S. Zhao, B. P. Sun, and W. M. Zhang, "Bifurcation and chaos analysis of a nonlinear electromechanical coupling relative rotation system," Chinese Physics B, vol. 23, no. 9, article 094501, 2014.

[14] S. Liu, H. Ai, Z. Pang, Z. Lin, and D. Zhao, "Hopf bifurcation control of nonlinear electromechanical coupling main drive system of rolling mill," The European Physical Journal Plus, vol. 135, no. 4, article 365, pp. 1-14, 2020.

[15] S. Liu, Y. K. Zhang, and B. Liu, "Dynamical bifurcation study on electromechanical coupling vibration in rolling mill's drive system," Journal of Mechanical Engineering, vol. 2, no. 46, p. 3, 2010, (in Chinese).

[16] S. Liu, S. S. Zhao, Z. L. Wang, and H. B. Li, "Stability and Hopf bifurcation of a nonlinear electromechanical coupling system with time delay feedback," Chinese Physics B, vol. 24, no. 1, article 014501, 2015.

[17] J. Wang, L. Ma, and Y. Wang, "Hopf bifurcation control for the main drive delay system of rolling mill," Advances in Difference Equations, vol. 2020, no. 1, Article ID 2666, 12 pages, 2020.

[18] Y. Ding, L. Zheng, and J. Xu, "Stability and bifurcation analysis of micro-electromechanical nonlinear coupling system with delay," Journal of Mathematical Analysis and Applications, vol. 461, no. 1, pp. 577-590, 2018.

[19] I. Petras, "Fractional-Order Nonlinear Systems: Modeling," in Analysis and Simulation, Springer, London, Beijing, 2011.

[20] I. Podlubny, Fractional Differential Equations, Academic Press, San Diego, 1999.

[21] S. G. Samko, A. A. Kilbas, and O. I. Marichev, Fractional Integrals and Derivatives Theory and Applications, Gordon and Breach, New York, NY, USA, 1993.

[22] Z. Li, D. Chen, J. Zhu, and Y. Liu, "Nonlinear dynamics of fractional order Duffing system," Chaos, Solitons and Fractals, vol. 81, pp. 111-116, 2015.

[23] J. Alidousti and E. Ghafari, "Dynamic behavior of a fractional order prey-predator model with group defense," Chaos, Solitons and Fractals, vol. 134, article 109688, 2020.

[24] S. Eshaghi, R. Khoshsiar Ghaziani, and A. Ansari, "Hopf bifurcation, chaos control and synchronization of a chaotic frac- tional- order system with chaos entanglement function," Mathematics and Computers in Simulation (MATCOM), vol. 172, pp. 321-340, 2020.

[25] B. Tao, M. Xiao, Q. Sun, and J. Cao, "Hopf bifurcation analysis of a delayed fractional-order genetic regulatory network model," Neurocomputing, vol. 275, pp. 677-686, 2018.

[26] M. X. Yi, L. F. Wang, and J. Huang, "Legendre wavelets method for the numerical solution of fractional integro- differential equations with weakly singular kernel," Applied Mathematical Modelling, vol. 40, no. 4, pp. 3422-3437, 2016.

[27] S. Eshaghi, R. K. Ghaziani, and A. Ansari, "Hopf bifurcation, chaos control and synchronization of a chaotic fractionalorder system with chaos entanglement function," Mathematics and computers in simulation, vol. 172, pp. 321-340, 2020.

[28] M. Xiao, G. Jiang, J. Cao, and W. Zheng, "Local bifurcation analysis of a delayed fractional-order dynamic model of dual congestion control algorithms," IEEE/CAA Journal of Automatica Sinica, vol. 4, no. 2, pp. 361-369, 2017.

[29] X. Liu and H. Fang, "Periodic pulse control of Hopf bifurcation in a fractional-order delay predator-prey model incorporating a prey refuge," Advances in Difference Equations, vol. 2019, no. 1, 30 pages, 2019.

[30] E. Ahmed, A. M. A. El-Sayed, and H. A. A. El-Saka, "On some Routh-Hurwitz conditions for fractional order differential equations and their applications in Lorenz, Rossler, Chua and Chen systems," Physics Letters A, vol. 358, no. 1, pp. 1-4, 2006.

[31] K. Diethelm, N. J. Ford, and A. D. Freed, "A predictorcorrector approach for the numerical solution of fractional differential equations," Nonlinear Dynamics, vol. 29, no. 1/4, pp. 3-22, 2002.

[32] K. Diethelm, "An algorithm for the numerical solution of differential equations of fractional order," Electronic transactions on numerical analysis, vol. 5, pp. 1-6, 1997.

[33] R. Garra and F. Polito, "Coupled systems of fractional equations related to sound propagation: analysis and discussion," Journal of Mathematical Physics, vol. 53, no. 4, article 043502, 2012.

[34] J. H. Jia, X. Y. Shen, and H. X. Hua, "Viscoelastic behavior analysis and application of the fractional derivative Maxwell model," Journal of Vibration and Control, vol. 13, no. 4, pp. 385-401, 2007.

[35] A. Shokooh and L. Suarez, "A comparison of numerical methods applied to a fractional model of damping materials," Journal of Vibration and Control, vol. 5, no. 3, pp. 331-354, 2007.

[36] J. Cao, C. Ma, Z. Jiang, and S. Liu, "Nonlinear dynamic analysis of fractional order rub-impact rotor system," Communications in Nonlinear Science and Numerical Simulation, vol. 16, no. 3, pp. 1443-1463, 2011.

[37] W. Chen, "Time-space fabric underlying anomalous diffusion," Chaos, Solitons and Fractals, vol. 28, no. 4, pp. 923929, 2005.

[38] J. H. He, "Fractal calculus and its geometrical explanation," Results in Physics, vol. 10, pp. 272-276, 2018.

[39] W. Cai, W. Chen, and W. Xu, "Characterizing the creep of viscoelastic materials by fractal derivative models," International Journal of Non-Linear Mechanics, vol. 87, pp. 58-63, 2016.

[40] J. Sabatier, O. P. Agrawal, and J. A. Tenreiro Machado, Advances in Fractional Calculus: Theoretical Developments and Applications in Physics and Engineering, Springer, 2007.

[41] T. Brezina and R. Jablonski, Mechatronics: recent technological and scientific advances, Springer, 2013. 